\title{
LINE PROFILE VARIATIONS IN AGN
}

\author{
W. KOLLATSCHNY and M. DIETRICH \\ Universitätssternwarte, Geismarlandstr. 11, D-37083 Göttingen, F.R.G.
}

An international collaboration is monitoring the variable Seyfert galaxy NGC 5548 in the optical spectral range since 1988. In Fig. 1 (left) the $\mathrm{H} \alpha$ light curves of the blue wing $\left(-6000\right.$ until $\left.-1000 \mathrm{~km} \mathrm{~s}^{-1}\right)$ and the red wing $(+1000$ until +6000 $\mathrm{km} \mathrm{s}^{-1}$ ) are shown for the first year of the monitoring campaign from Dec.1988 until Oct.1989. It can be seen that these line components of the $\mathrm{H} \alpha$ profile have different amplitudes in the light curves. The mean $\mathrm{H} \alpha$ and $\mathrm{H} \beta$ difference spectra with respect to the minimum state are plotted in Fig. 1 (right) for the same period. The relative strength of the blue component at $\mathrm{v}_{\mathrm{rel}}=-2000 \mathrm{~km} \mathrm{~s}^{-1}$ is different with respect to the core of the line profiles. Therefore, these components originate under different physical conditions or in regions with different dust content.

This work has been supported by DFG grant Ko 857/13-1
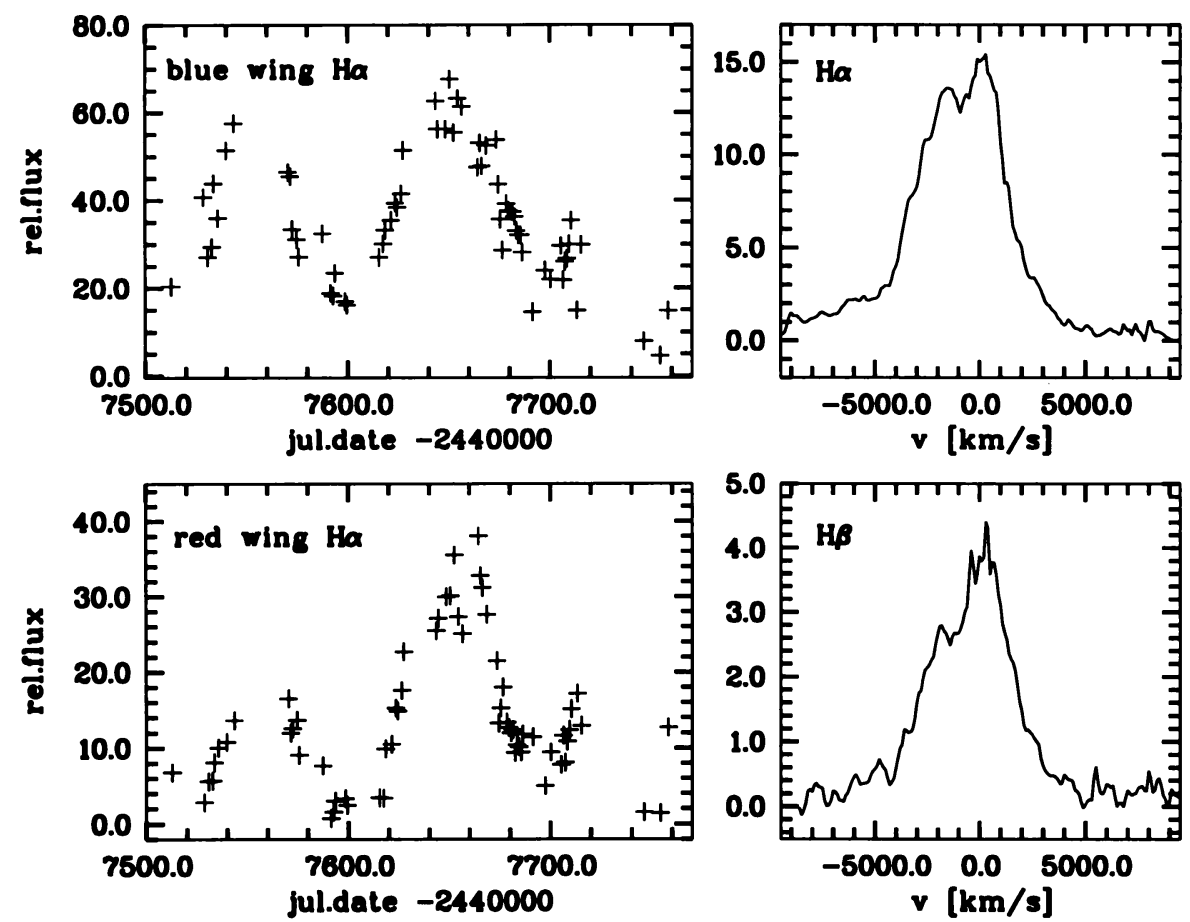

Fig. 1. Light curve of the blue and red wing of the $\mathrm{H} \alpha$ emission line (left). Mean difference spectra of the $\mathrm{H} \alpha$ and $\mathrm{H} \beta$ emission lines for the first year of the monitoring campaign of NGC 5548 (right).

395

T. J.-L. Courvoisier and A. Blecha: Multi-Wavelength Continuum Emission of AGN, 395.

(C) 1994 IAU. Printed in the Netherlands. 